\title{
Alternative Conservative Approach for Tooth Build- Up in Patients with Special Healthcare Needs
}

\author{
Mayank Kakkar ${ }^{1}$, Vijay Rahul Markonda Patnaik ${ }^{2}$, Shaima Malik ${ }^{3}$, Shilpa Singh ${ }^{4}$, Adela Planerova ${ }^{5}$ \\ ${ }^{1}$ Dental Resident, Department of General Dentistry, Eastman Institute for Oral Health, University of Rochester, \\ New York, USA \\ ${ }^{2}$ Dental Preceptee, Department of General Dentistry, Eastman Institute for Oral Health, University of Rochester, \\ New York, USA \\ ${ }^{3}$ Department of Orthodontics and Dentofacial Orthopedics, Eastman Institute for Oral Health, Rochester, \\ New York, USA \\ ${ }^{4}$ Department of Orofacial Pain/TMD and Community Dentistry, Eastman Institute for Oral Health, Rochester, \\ New York, USA \\ ${ }^{5}$ Department of General Dentistry, Eastman Institute for Oral Health, University of Rochester, NY, USA
}

*Corresponding Author: Mayank kakkar; mayankuever@gmail.com

Received 18 August 2021;

Accepted 11 September 2021;

Published 16 September 2021

\begin{abstract}
Purpose: The success of an artificial crown mainly depends on the biomechanical factors such as bonding capacity of cement between the crown and abutment tooth, and root strength of the abutment tooth. In this case series, we aim to provide insight on the potential use of the cast metal post and cost for patients with intellectual disabilities and severely compromised tooth structure. Material and Methods: The first patient was a 41-year old male with the chief complaint of a dislodged porcelain fused metal (PFM) crown of tooth \#20 (mandibular left second bicuspid). The patient was informed about the advantages, disadvantages, and risks of each option. After further consideration and discussion with the parents and the patient, we decided to rebuild the coronal tooth structure with the cast metal post and core and prosthetic restoration with the same crown. Patient satisfaction was measured on the visual analog scale as 10/10. The second case involves a 26-year old female, who presented with a chief complaint of chronic lingering pain on tooth \#9. The tooth structure was extensively damaged and un-supportive of the ferrule, therefore, it was decided to build a metal post and core instead of a fiber post. The prosthetic restoration was made with a monolithic zirconia crown and the patient was followed up twice in 6 months and graded the satisfaction as 10/10 on a visual analog scale. Results: The most common type of posts used in dentistry has been cast post and cores in the last decade which generally includes an additional laboratory stage where a custom post is prepared according to the impression taken from the prepared post space. Conclusions: Both the presented cases explain an interesting advantage of restoring a root canal treated tooth with a metal post and core system which implies that in a prefabricated post and core restored tooth failure, a metal post, and core can potentially be the treatment of choice instead of extracting the tooth.
\end{abstract}

\section{Keywords: Post and core, Special needs, Cast metal Post}

\section{Introduction}

The success of an artificial crown mainly depends on the biomechanical factors such as bonding capacity of cement between the crown and abutment tooth, root strength of the abutment tooth. Most of the crown restoration failures occur due to poor root strength of the abutments leading to fractures along the root or at a fracture line just above the supra-ferrule margin.

\section{Background}

For more than two centuries the researchers have documented the use of post and core to restore the functionality and esthetics of a WWW.ijirms.in tooth ${ }^{[1]}$. Pierre Fauchard, 1728 used the tenons, which are metal posts screwed into the roots ${ }^{[1]}$. Later in 1800 's the wooden posts were commonly used among the dentists where the wooden post is used to pivot the crown, and the post is fitted into the root of the tooth ${ }^{[1]}$. Wooden posts have major disadvantage of water absorption that may compromise the strength resulting in root fracture. In the 19th century, Richmond- crowns were the new strategy to support the prosthetic crowns in the root canal treated tooth with less tooth material. It is a one-piece post crown and it can be bent in any direction without breaking and maintain the shape rigidity ${ }^{[2]}$. In 1930s, the Richmond one-piece crowns were replaced by the present days metal post and core which were casted as a separate component ${ }^{[3]}$. The newer technique involves better 
margin adaptation for variation in the path of insertion. Cast metal post-core systems have a long history of success use because of their superior physical properties ${ }^{[4]}$. Custom-fabricated cast gold post and core has been used for decades as a foundation restoration to support the final restoration in endodontic treated teeth. A retrospective study reported a success rate of 90.6 percent using a cast post and core as a foundation restoration ${ }^{[5]}$. Because esthetics was a major disadvantage with the metal post and core, use of porcelain fused to metal post and core from a metal ceramic alloy to mask the shade of the metal became more common ${ }^{[6]}$. A successful post and core restoration of endodontic tooth depends on ferrule effect and preservation of cavity walls ${ }^{[7]}$.

The ferrule effect occurs when a ferrule, or a metal or porcelain band, is cemented 360 degrees around a tooth or an abutment and binds the tooth and/or core and/or post structures that exist superior to the ferrule margin. This binding results in two "effects." First, the ferrule prevents independent flexure of the different components of these supra-ferrule-margin structures in response to occlusal forces, which would normally occur if the structures were not ferruled. Second, the ferrule has the effect of transferring the occlusal forces from these supra-ferrule-margin structures to the margin of the ferrule ${ }^{[8]}$. Ferrule effect causes occlusal forces applying energy to a variety of different stress planes within the supra-gingival tooth/core/post structures of the non-ferruled tooth, the occlusal force energy is transferred by the ferrule to the cross sectional area or the plane of tooth/core/post structure that exists approximately and theoretically at the level of the ferrule margin ${ }^{[9]}$. The ferrule tooth structure is approximately $1.5-2 \mathrm{~mm}$ above the ferrule margin ${ }^{[10,11]}$. The total bonding strength at the cross sectional area of the ferrule tooth complex, is a sum of the respective bond strengths of tooth structure, core structure, and post structure that molecularly bond the sub-ferrulemargin tooth/core/post complex with the supra ferrule-margin tooth/core/post complex ${ }^{[9]}$.

This paper will present two cases with the aim to provide insight on the potential use of the cast metal post, the costeffectiveness, and the ease of use in the patients with special needs and severely compromised tooth structure.

\section{\#1 Case Presentation}

A 41 years old male patient presented with the chief complaint of a dislodged porcelain fused metal (PFM) crown on tooth \#20 (mandibular left second bicuspid). A comprehensive medical history evaluation revealed developmental disorder, childhood seizures, and anxiety. The previous dental treatment was conducted in a private dental practice. Tooth \#20 was root canal treated because of the deep caries lesion on the mesio-occlusal surfaces of the tooth. Later, it was reconstructed with a fiber-post and core system and a PFM crown. Clinical intraoral examination suggested a poorly retained crown for tooth \# 20 and increased sensitivity to vertical percussion (visual analogue scale- 7/10). Patient's gingival status was within the normal limits with no gingival inflammation or bleeding on probing. Intraoral periapical radiographic examination showed enough osseous support to the root, and the ferrule margin nearly $2 \mathrm{~mm}$ when measured around the tooth. After complete clinical and radiological evaluation, three different treatment modalities were discussed with the patient, 1) rebuilding cast metal post and core, 2) Extraction of \#20 and restoration with crowns and bridges, 3) extraction followed by dental implant restoration, 4) redoing the buildup with fiber post and new crown. The advantages, disadvantages and risk of each treatment option were discussed in detail. Patient opted for the rebuilding the coronal tooth structure with the cast metal post and core and prosthetic restoration using the same crown. During the first visit, post space was created with the slow hand piece and the impression was taken with the Polyvinyl siloxane (PVS) light and heavy body impression material. The impression was sent to the lab along with the existing crown. Detailed discussion with the lab technician was done from time to time and progress was monitored. After 2 weeks, patient was scheduled for an appointment. The crown internal fitting and the occlusion was checked and after making sure everything was adequate, cast metal post and crown was cemented with the resin based dual core cement material. High points were checked with the articulating paper. Patient was followed up after 2 weeks of crown placement, thereafter; follow-up visits were scheduled at 3 months, and 6months. Clinical and radio graphical findings suggested good periodontal condition and no pain was reported. Patient satisfaction was measured on the visual analogue scale as 10/10.

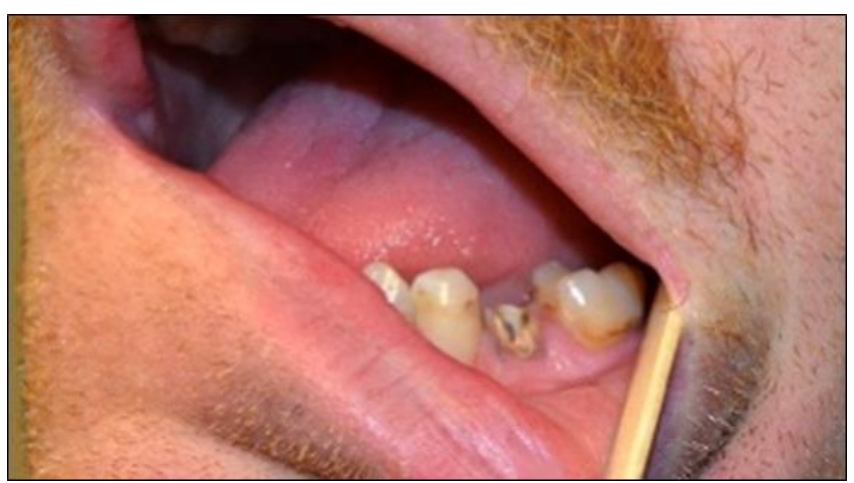

Figure 1: Pre-operative picture of teeth \#20 which had previous RCT

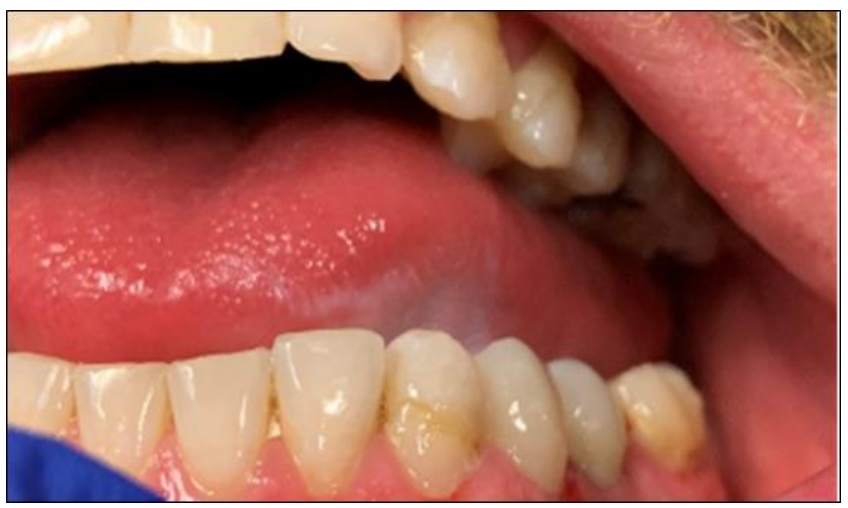

Figure 2: Post-operative picture of Re-treated RCT with cast post and core with final full coverage crown.

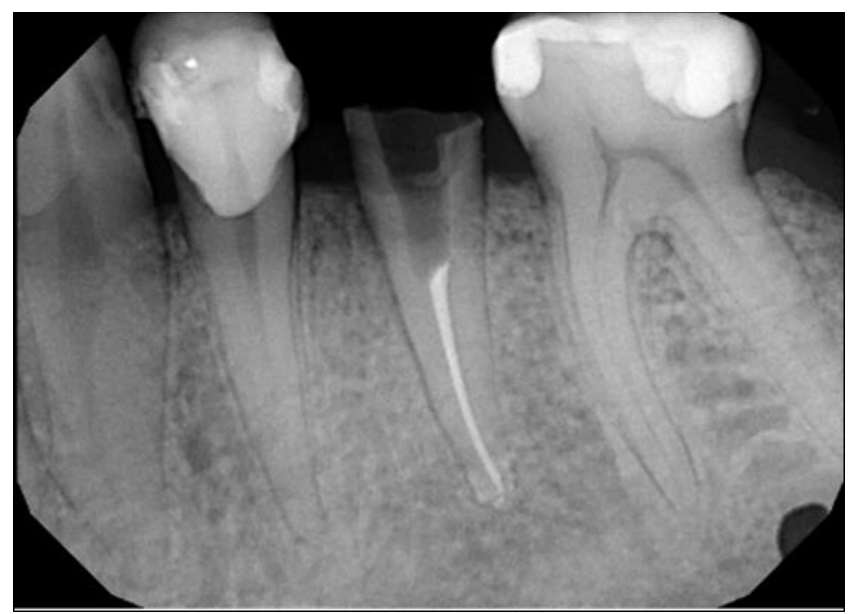

Figure 3: Radiograph with tooth \#20 with preparation for post and core. 


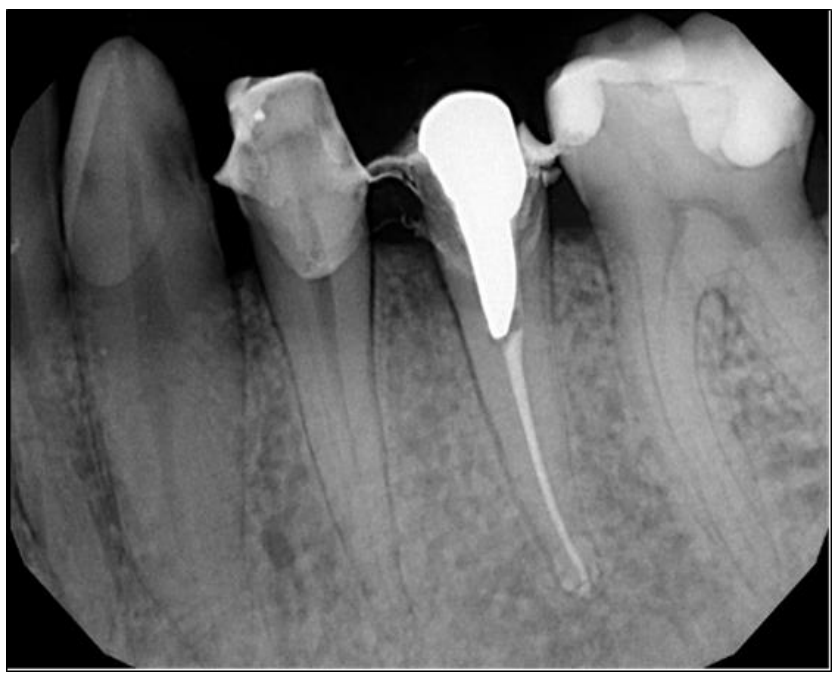

Figure 4: Radiograph with tooth \#20 with restoration of a cast post and core

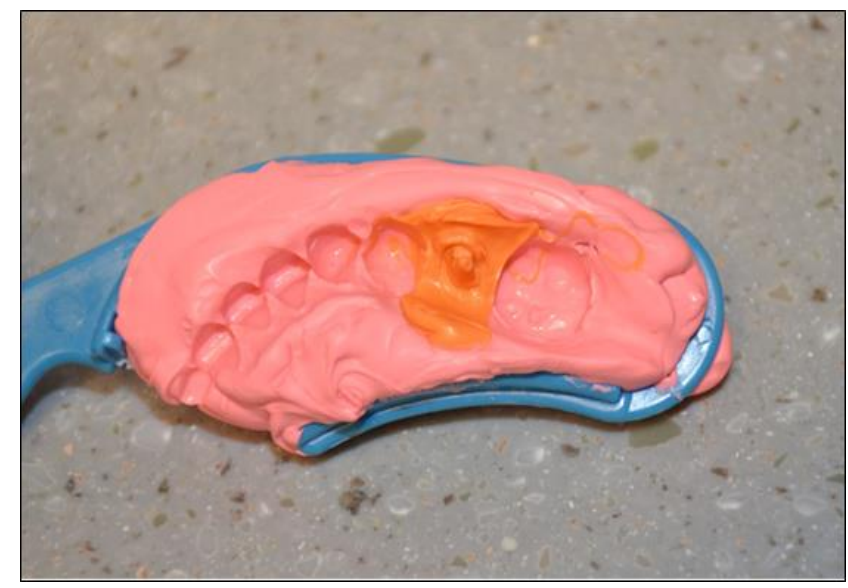

Figure 5: Impression made with PVS light and heavy body impression material for designing the post and core.

\section{\#2 Case Presentation}

This clinical case illustrates the potential implication of custom metal post and core system in restoring a tooth which is severely compromised. A 26-year-old female presented with a chief complaint of chronic lingering pain on tooth \#9. Patient reported spontaneous onset of pain that worsened with hot and cold drinks. The pain was recorded 7/10 on a visual analogue scale. Medical history was reviewed and revealed history of systemic lupus erythematous, thrombocytopenia, GERD, Asthma, and Rheumatic juvenile arthritis. Intraoral and radiographic examination showed caries on tooth \#9 approaching the pulp and esthetic impairment related to the same tooth.

Percussion test was negative ruling out acute apical periodontitis. A hot test using hot water in a Monojet syringe and rubber dam isolation, was performed, to reproduce pain. Healthy control tooth was also tested first. Delayed and prolonged pain triggered by heat could be indicative of irreversible pulpitis. Radiographic examination confirmed the deep carious lesion involving enamel dentin and pulp. After ruling out nonodontogenic pain; musculoskeletal, neurovascular, and neuropathic pain or any other underlying pathologies, we diagnosed her with acute irreversible pulpitis of \#9 with normal apical periodontitis The gingival status of the patient was found to have mild generalized inflammation of gingiva.

The tooth structure was extensively damaged and unsupportive of ferrule, therefore, it was decided to build the metal post and core instead of fiber post. The shoulder margin was to deep, so we had to perform gingivectomy along the circumference of the tooth and also performed crown lengthening. Prosthetic restoration was made with a monolithic zirconia crown and patient was followed up twice in 6 months and graded the satisfaction as $10 / 10$ on a visual analogue scale.

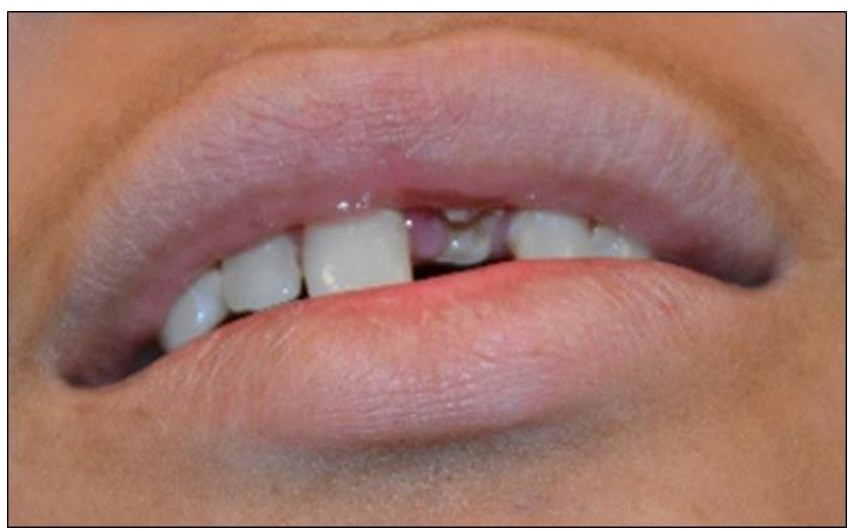

Figure 6: Pre-operative picture of a \#9 which is previous RCT that subsequently failed.

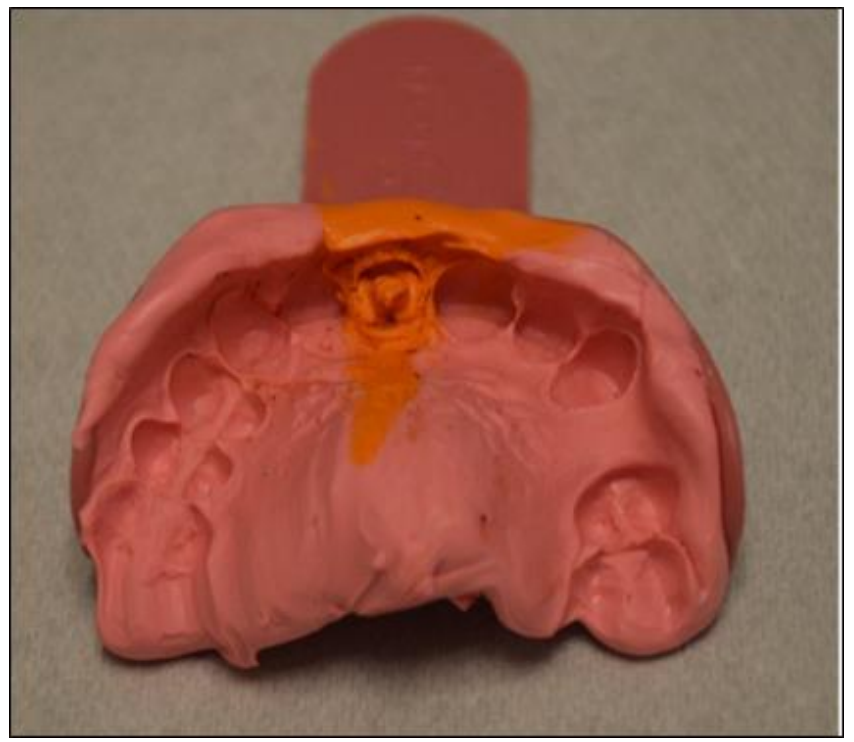

Figure 7: Impression made with PVS light and heavy body impression material for designing the post and core.

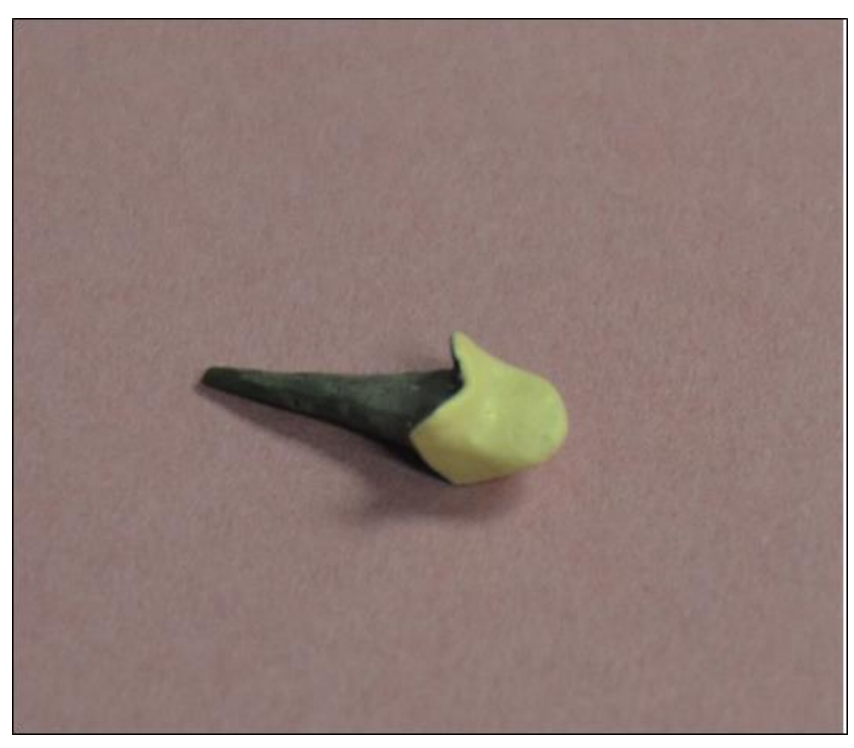

Figure 8: Image of a cast/customized metal Post and core 


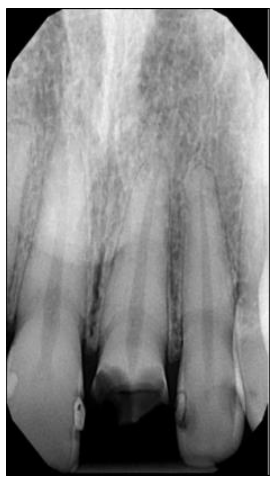

(a)

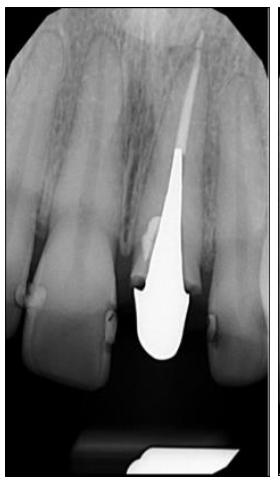

(b)

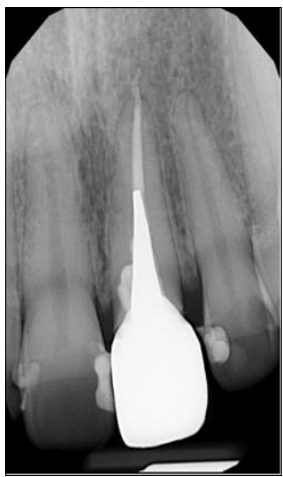

(c)
Figure 9: a) Radiograph with tooth \#9 Pre-op. b) Radiograph with \#9 with restoration of a cast post and core. c) Radiograph with PFM crown restored on the cast post and core.

\section{Discussion}

Intra-radicular posts are useful adjuncts in the restoration of endodontic treated teeth for more than two centuries. These systems have undergone a significant evolution in recent years, and fiber-reinforced prefabricated systems and metal post-and coresystems have started to be incorporated into routine clinical care. It is generally the common opinion of dental authorities that endodontically treated teeth are more prone to fracture due to a variety of factors such as extensive tissue loss, loss of moisture content, and flexibility as well as decrease in resistance due to endodontic access preparations ${ }^{[12]}$. The most common type of posts used in dentistry has been cast post and cores in the last decade which generally includes an additional laboratory stage where a custom post is prepared according to the impression taken from the prepared post space ${ }^{[13]}$. As dentists our goal is to preserve the integrity of natural teeth. Both cases presented here highlight an interesting advantage of restoring a root canal treated tooth with metal post and core system which implies that in a prefabricated post and core restored tooth failure, a metal post and core can potentially be the treatment of choice instead of extracting the tooth. Both patients were special needs patients. In case \#1, the patient was not cooperative and had dental phobia and had requested as to extract tooth \#20. We recommended to restore it with custom metal post and core and use his previously fabricated crown to restore which was would be economical for him, we were able to convince the patient to restore the tooth $\# 20$ with the previously fabricated porcelain-fused to metal (PFM) crown on the metal post and core.

\section{Ethics statement}

There was no human or animals participants involved. There was no human data that was collected.

\section{Conflict of interest statement}

No conflict of interests

\section{Acknowledgement}

There is no financial support involved with this research.

\section{References}

[1] Smith, C.T., N.J. Schuman, and W. Wasson, Biomechanical criteria for evaluating prefabricated post-and-core systems: a guide for the restorative dentist. Quintessence Int, 1998. 29(5): p. 305-12.

[2] Tamarin, A.H., A New Type of Richmond Crown. J Am Dent Assoc, 1964. 69: p. 557-9.

[3] Smith, C.T. and N. Schuman, Prefabricated post-andcore systems: an overview. Compend Contin Educ Dent, 1998. 19(10): p. 1013-8, 1020; quiz 1022.

[4] Ozkurt, Z., U. Iseri, and E. Kazazoglu, Zirconia ceramic post systems: a literature review and a case report. Dent Mater J, 2010. 29(3): p. 233-45.

[5] Bergman, B., et al., Restorative and endodontic results after treatment with cast posts and cores. J Prosthet Dent, 1989. 61(1): p. 10-5.

[6] Cheung, W., A review of the management of endodontically treated teeth. Post, core and the final restoration. J Am Dent Assoc, 2005. 136(5): p. 611-9.

[7] Naumann, M., et al., "Ferrule Comes First. Post Is Second!" Fake News and Alternative Facts? A Systematic Review. J Endod, 2018. 44(2): p. 212-219.

[8] Ha, S.R., Biomechanical three-dimensional finite element analysis of monolithic zirconia crown with different cement type. J Adv Prosthodont, 2015. 7(6): p. 475-83.

[9] Mamoun, J.S., On the ferrule effect and the biomechanical stability of teeth restored with cores, posts, and crowns. Eur J Dent, 2014. 8(2): p. 281-286.

[10] Tjan, A.H. and S.B. Whang, Resistance to root fracture of dowel channels with various thicknesses of buccal dentin walls. J Prosthet Dent, 1985. 53(4): p. 496-500.

[11] McLean, A., Criteria for the predictably restorable endodontically treated tooth. J Can Dent Assoc, 1998. 64(9): p. 652-6.

[12] Monga P, Sharma V, Kumar S. Comparison of fracture resistance of endodontically treated teeth using different coronal restorative materials: An in vitro study. J Conserv Dent. 2009 Oct;12(4):154-9. doi: 10.4103/09720707.58338. PMID: 20543925; PMCID: PMC2879728.

[13] An Overview of Clinical Studies on Fiber Post Systems Idil Dikbas and Jale Tanalp ScientificWorldJournal. 2013; 2013: 171380. Published online 2013 Oct 23. doi: $10.1155 / 2013 / 171380$ 